Tôhoku Math. Journ.

30 (1978), 575-581.

\title{
ON ABSTRACT MEAN ERGODIC THEOREMS
}

\author{
RYOTARO SATO
}

(Received May 23, 1977)

1. Introduction. In [8] Sine showed an interesting mean ergodic theorem. His theorem states that the ergodic averages $(1 / n) \sum_{i=0}^{n-1} T^{i}$ converge in the strong operator topology if and only if the fixed points of $T$ separate the fixed points of the adjoint operator $T^{*}, T$ being any linear contraction on a Banach space. Later, this theorem was generalized and extended by Nagel [5] to a bounded right amenable operator semigroup in a Banach space. Another generalization was also done by Lloyd [4]. In the present paper we intend to apply the notion of "ergodicity", given for an operator semigroup in a locally convex topological vector space and originally introduced by Eberlein [3], and obtain abstract mean ergodic theorems which generalize Sine's, Nagel's and Lloyd's ergodic theorems.

2. Definitions and examples. Throughout this paper, $E$ will denote a complete locally convex topological vector space (t. v. s.) and $\mathfrak{S}$ a semigroup of continuous linear operators on $E$. For $x \in E$ we denote by $A(x)$ the affine subspace of $E$ determined by the set $\{T x: T \in \mathbb{S}\}$, i.e.,

$$
A(x)=\left\{y: y=\sum_{i=1}^{n} a_{i} T_{i} x, \sum_{i=1}^{n} a_{i}=1, T_{i} \in \mathfrak{S}, 1 \leqq n<\infty\right\},
$$

and by $\bar{A}(x)$ the closure of $A(x)$ in $E$. Let $\left(T_{\alpha}, \alpha \in \Lambda\right)$ be a net of linear operators on $E . \quad\left(T_{\alpha}, \alpha \in \Lambda\right)$ is said to be a (weakly) right [resp. (weakly) left] S-ergodic net if it satisfies:

(I ) For every $x \in E$ and all $\alpha \in \Lambda, T_{\alpha} x \in \bar{A}(x)$.

(II) The transformations $T_{\alpha}$ are equicontinuous.

(III) For every $x \in E$ and all $T \in \mathfrak{S}$,

$$
\text { (weak-) } \left.\lim _{\alpha} T_{\alpha} T x-T_{\alpha} x=0 \text { [resp. (weak-) } \lim _{\alpha} T T_{\alpha} x-T_{\alpha} x=0\right] \text {. }
$$

S is said to be a (weakly) right [resp. (weakly) left] ergodic semigroup (in the sense of Eberlein [3]) if it possesses at least one (weakly) right [resp. (weakly) left] S-ergodic net $\left(T_{\alpha}, \alpha \in \Lambda\right)$. Whenever $\left(T_{\alpha}, \alpha \in \Lambda\right)$ is a (weakly) right and left both S-ergodic net, we call it simply a (weakly) S-ergodic net. And if $\mathfrak{S}$ possesses at least one (weakly) $\mathfrak{S}$-ergodic net 
$\left(T_{\alpha}, \alpha \in \Lambda\right), \mathfrak{S}$ is said to be a (weakly) ergodic semigroup. (See also Day [1].) Here we note that our definition of ergodicity is somewhat different from that of Eberlein [3]. Instead of our condition (I), he used the following stronger condition:

(S-I) For every $x \in E$ and all $\alpha \in \Lambda, T_{\alpha} x \in \overline{\operatorname{co}} \subseteq x$, where $\overline{c o} \subseteq x$ denotes the closed convex hull of the set $\{T x: T \in \mathfrak{S}\}$.

Examples. (1) Suppose either (i) $0 \in \mathfrak{S}$ or (ii) $\lambda_{i} I \in \mathfrak{S}(i=1,2)$ with $\lambda_{1} \neq \lambda_{2}$, $I$ being the identity operator. It follows, in either case, that $0 \in \bar{A}(x)$ for every $x \in E$. Thus the sequence $\left(T_{n}, n \geqq 1\right)$, defined by $T_{n}=$ 0 for all $n \geqq 1$, is an S-ergodic net.

(2) Suppose $T$ is a bounded linear operator of spectral radius $r(T) \leqq$ 1 on a Banach space. If $\sup _{0<r<1}\left\|(1-r) \sum_{n=0}^{\infty} r^{n} T^{n}\right\|<\infty$, then $\mathfrak{S}=$ $\left\{T^{n}: n \geqq 0\right\}$ is an ergodic semigroup. In fact, putting $T_{r}=(1-r) \sum_{n=0}^{\infty} r^{n} T^{n}$ $(0<r<1)$, we have an S-ergodic net $\left(T_{r}, 0<r<1\right)$.

(3) Let $C(\mathfrak{S})$ denote the space of all bounded continuous functions on $\mathfrak{S}, \mathfrak{S}$ being equipped with the weak operator topology. It is then easily seen that, for each $f$ in $C(\mathfrak{S})$ and each $S$ in $\mathfrak{S},{ }_{s} f$ and $f_{S}$ are again in $C(\mathfrak{S})$, where ${ }_{s} f$ and $f_{S}$ are defined by

$$
{ }_{s} f(T)=f(S T) \text { and } f_{S}(T)=f(T S) \quad(T \in \mathfrak{S}) .
$$

A linear functional $\mu$ on $C(\mathfrak{S})$ is said to be a right [resp. left] invariant mean if $\mu$ satisfies $\|\mu\|=1=\langle 1, \mu\rangle$ and $\left\langle f_{s}, \mu\right\rangle=\langle f, \mu\rangle\left[\operatorname{resp} .\left\langle{ }_{s} f, \mu\right\rangle=\right.$ $\langle f, \mu\rangle]$ for every $S \in \mathfrak{S}$ and all $f \in C(\mathfrak{S})$.

Proposition 1. Suppose $\subseteq$ is an equicontinuous semigroup of linear operators on $E$. If there exists a right [resp. left] invariant mean on $C(\mathfrak{S})$, then $\mathfrak{S}$ is a weakly right [resp. weakly left] ergodic semigroup.

Proof. Let $l_{1}(\mathfrak{S})$ denote the space of all functions $\xi$ defined on $\mathfrak{S}$ for which the norm is given by

$$
\|\xi\|_{1}=\sum_{S \in \mathbb{S}}|\xi(S)|<\infty .
$$

From Section 10 of Day [2] it follows that if $C(\Im)$ has a right [resp. left] invariant mean, then there exists a net $\left(\xi_{\alpha}, \alpha \in \Lambda\right)$ of elements in $l_{1}(\mathfrak{S})$ such that

(i ) for every $\alpha \in \Lambda, \xi_{\alpha} \geqq 0$ on $\mathfrak{S}, \sum_{S_{\in} \subseteq} \xi_{\alpha}(S)=1$ and $\left\{S \in \mathfrak{S}: \xi_{\alpha}(S)>\right.$ $0\}$ is a finite set,

(ii) for every $f \in C(\mathfrak{S})$ and all $T \in \mathfrak{S}$, $\lim _{\alpha} \sum_{S \in \mathbb{S}} f(S)\left(\xi_{\alpha} * \delta_{T}(S)-\xi_{\alpha}(S)\right)=0 .\left[\operatorname{resp} . \lim _{\alpha} \sum_{S \in \mathbb{S}} f(S)\left(\delta_{T} * \xi_{\alpha}(S)-\xi_{\alpha}(S)\right)=0\right]$, 
where $\xi_{\alpha} * \delta_{T}$ and $\delta_{T} * \xi_{\alpha}\left(\in l_{1}(\mathfrak{S})\right)$ are defined by

$$
\xi_{\alpha} * \delta_{T}(S)=\sum_{R T=S} \xi_{\alpha}(R) \text { and } \delta_{T} * \xi_{\alpha}(S)=\sum_{T R=S} \xi_{\alpha}(R)
$$

for all $S \in \mathbb{S}$.

Let us put $T_{\alpha}=\sum_{S \in \Im} \xi_{\alpha}(S) S$. We shall prove that the net $\left(T_{\alpha}, \alpha \in\right.$ A) is a weakly right [resp. weakly left] S-ergodic net.

To do this, fix an $x \in E$ and an $x^{*} \in E^{*}$ arbitrarily, $E^{*}$ being the dual space of $E$, and define a function $f$ on $\mathfrak{S}$ by the relation: $f(S)=$ $\left\langle S x, x^{*}\right\rangle$ for all $S \in \mathfrak{S}$. Since $\mathfrak{S}$ is equicontinuous, $f$ is a bounded function on $\mathfrak{S}$, and hence $f$ is in $C(\mathfrak{S})$. It follows that for all $T \in \mathfrak{S}$,

$$
\begin{gathered}
\lim _{\alpha}\left\langle T_{\alpha} T x-T_{\alpha} x, x^{*}\right\rangle=\lim _{\alpha} \sum_{S \in \mathbb{S}} f(S)\left(\xi_{\alpha} * \delta_{T}(S)-\xi_{\alpha}(S)\right) \\
=0\left[\text { resp. } \lim _{\alpha}\left\langle T T_{\alpha} x-T_{\alpha} x, x^{*}\right\rangle=0\right],
\end{gathered}
$$

and therefore we have weak- $\lim _{\alpha} T_{\alpha} T x-T_{\alpha} x=0$ [resp. weak-lim ${ }_{\alpha} T T_{\alpha} x-$ $\left.T_{\alpha} x=0\right]$. Clearly, $\left(T_{\alpha}, \alpha \in \Lambda\right)$ satisfies conditions (S-I) and (II). The proof is complete.

\section{Abstract mean ergodic theorems.}

THEOREM 1. Let $E$ be a complete locally convex t.v.s. and $\subseteq$ a semigroup of continuous linear operators on $E$. Suppose $\left(T_{\alpha}, \alpha \in \Lambda\right)$ is a weakly right S-ergodic net, and define

$$
D=\left\{x \in E: \text { weak- } \lim _{\alpha} T_{\alpha} x \text { exists }\right\}
$$

and

$$
T_{\infty} x=\text { weak- } \lim _{\alpha} T_{\alpha} x \quad(x \in D)
$$

Then we have:

(a) $D$ is a closed linear subspace of $E$ such that $T D \subset D$ for all $T \in$ ऽ.

(b) $T_{\infty} D \subset D$ and $T_{\infty}$ is linear and continuous on $D$.

(c) $T_{\infty} T=T_{\infty}$ on $D$ for all $T \in \mathfrak{S}$.

Proof. It is easily seen that $D$ is a linear subspace of $E$. Since $\left(T_{\alpha}, \alpha \in \Lambda\right)$ is a weakly right S-ergodic net, it follows that $T D \subset D$ and $T_{\infty} T=T_{\infty}$ for all $T \in \mathfrak{S}$. Thus (c) is proved. To see that $D$ is closed, we show that $D$ is complete. Let $\left(x_{\beta}\right)$ be a Cauchy net in $D$. Since the transformations $T_{\alpha}$ are equicontinuous, $\left(T_{\infty} x_{\beta}\right)$ is also a Cauchy net in $E$. Thus if we let 


$$
x=\lim _{\beta} x_{\beta} \text { and } y=\lim _{\beta} T_{\infty} x_{\beta},
$$

then, given a weak convex neighborhood $U$ of $0 \in E$, there exists a $\beta_{0}$ such that

$$
T_{\infty} x_{\beta_{0}}-y \in(1 / 3) U \text { and } T_{\alpha}\left(x-x_{\beta_{0}}\right) \in(1 / 3) U
$$

for all $\alpha \in \Lambda$. Since $T_{\infty} x_{\beta_{0}}=$ weak- $\lim _{\alpha} T_{\alpha} x_{\beta_{0}}$, there exists an $\alpha_{0}$ such that

$$
T_{\alpha} x_{\beta_{0}}-T_{\infty} x_{\beta_{0}} \in(1 / 3) U
$$

for all $\alpha \in \Lambda$ with $\alpha>\alpha_{0}$. Then, for all $\alpha \in \Lambda$ with $\alpha>\alpha_{0}$, we have

$$
\begin{gathered}
T_{\alpha} x-y=T_{\alpha}\left(x-x_{\beta_{0}}\right)+T_{\alpha} x_{\beta_{0}}-T_{\infty} x_{\beta_{0}}+T_{\infty} x_{\beta_{0}}-y \\
\in(1 / 3) U+(1 / 3) U+(1 / 3) U=U,
\end{gathered}
$$

so that $y=$ weak- $\lim _{\alpha} T_{\alpha} x$ and $x \in D$. Thus (a) is proved. By (a), $T_{\alpha} x \in$ $\bar{A}(x) \subset D$ for all $x \in D$ and all $\alpha \in A$, and this implies that $T_{\infty} D \subset D$. It is easily seen that $T_{\infty}$ is linear and continuous on $D$. The proof is complete.

From now on we shall always assume that $\left(T_{\alpha}, \alpha \in \Lambda\right)$ is a weakly right $\subseteq$-ergodic net, unless the contrary is explicitly specified. Let $\mathfrak{\Im}^{*}=$ $\left\{T^{*}: T \in \mathfrak{S}\right\}$ denote the adjoint semigroup of $\mathfrak{S}$. Define

$$
\begin{aligned}
F & =\{x \in E: T x=x \text { for all } T \in \mathfrak{S}\}, \\
D(F) & =\left\{x \in D: T_{\infty} x \in F\right\}, \\
D(0) & =\left\{x \in D: T_{\infty} x=0\right\},
\end{aligned}
$$

and

$$
F^{*}=\left\{x^{*} \in E^{*}: T^{*} x^{*}=x^{*} \text { for all } T^{*} \in \mathfrak{S}^{*}\right\} .
$$

Then we have

THEOREM 2.

(a) $F$ and $D(F)$ are closed linear subspaces of $E$ such that $F \subset$ $D(F) \subset D$.

(b) $T_{\infty} D(F) \subset D(F)$ and $T D(F) \subset D(F)$ for all $T \in \mathbb{S}$.

(c) $T T_{\infty}=T_{\infty} T=T_{\infty}$ on $D(F)$ for all $T \in \mathfrak{S}$.

(d) $D(0)$ is the closed linear subspace of $E$ determined by the set $\{x-T x: x \in E$ and $T \in \mathfrak{S}\}$.

(e) $y \in \bar{A}(x) \cap F$ if and only if $x \in D(F)$ and $y=T_{\infty} x$.

Proof. Since (a), (b), and (c) are direct from Theorem 1, we omit the details.

To prove (d), we first notice that $D(0)$ is a closed linear subspace 
of $E$. If we denote by $N$ the closed linear subspace of $E$ determined by the set $\{x-T x: x \in E$ and $T \in \mathcal{S}\}$, then $N \subset D(0)$, since weak- $\lim _{\alpha}$ $T_{\alpha} T x-T_{\alpha} x=0$ for every $x \in E$ and all $T \in \mathbb{S}$. Now suppose $x_{0} \in D(0)$. If $x^{*} \in E^{*}$ satisfies $\left\langle y, x^{*}\right\rangle=0$ for all $y \in N$, then $x^{*} \in F^{*}$, because $\langle x-$ $\left.T x, x^{*}\right\rangle=0$ for every $x \in E$ and all $T \in S$. Thus

$$
\left\langle x_{0}, x^{*}\right\rangle=\left\langle T_{\infty} x_{0}, x^{*}\right\rangle=\left\langle 0, x^{*}\right\rangle=0,
$$

because $T_{\infty} x_{0} \in \bar{A}\left(x_{0}\right)$. Hence, by the separation theorem (see, for example, Theorem 3.5 of [6]), $x_{0} \in N$. This proves (d).

To prove (e), let $y \in \bar{A}(x) \cap F$. Given a weak convex and balanced neighborhood $U$ of $0 \in E$, there exists a neighborhood $V$ of $0 \in E$ (in the topology originally given in $E$ ) such that

$$
T_{\alpha} V \subset(1 / 2) U
$$

for all $\alpha \in \Lambda$, since the transformations $T_{\alpha}$ are equicontinuous. Choose $\sum_{i=1}^{n} a_{i} T_{i} x \in A(x)$ so that

$$
y-\sum_{i=1}^{n} a_{i} T_{i} x \in V .
$$

Since $\left(T_{\alpha}, \alpha \in \Lambda\right)$ is a weakly right $S_{-e r g o d i c}$ net, then there exists an $\alpha_{0}$ such that

$$
T_{\alpha} T_{i} x-T_{\alpha} x \in\left(1 / 2 \sum_{i=1}^{n}\left|a_{i}\right|\right) U
$$

for all $i=1, \cdots, n$ and all $\alpha \in \Lambda$ with $\alpha>\alpha_{0}$. Then, for all $\alpha \in \Lambda$ with $\alpha>\alpha_{0}$,

$$
\begin{aligned}
y-T_{\alpha} x= & T_{\alpha} y-T_{\alpha} x \in T_{\alpha}\left(\sum_{i=1}^{n} a_{i}\left(T_{i} x-x\right)\right) \\
& +T_{\alpha} V \in \sum_{i=1}^{n} a_{i}\left(1 / 2 \sum_{i=1}^{n}\left|a_{i}\right|\right) U+(1 / 2) U=U,
\end{aligned}
$$

thus $y=$ weak- $\lim _{\alpha} T_{\alpha} x$ and $x \in D(F)$. The converse implication is obvious. The proof is complete.

THEOREM 3. $C$ is a closed linear subspace of $T_{\infty} D$ and separates $F^{*}$ if and only if $T_{\infty} D=C$ and $D=E$.

Proof. First suppose that $C$ is a closed linear subspace of $T_{\infty} D$ and separates $F^{*}$. Write

$$
D(C)=\left\{x \in D: T_{\infty} x \in C\right\} .
$$

$D(C)$ is a closed linear subspace of $D$, and thus it is also a closed linear subspace of $E$. Let $x^{*} \in E^{*}$ be such that $\left\langle x, x^{*}\right\rangle=0$ for all $x \in D(C)$. 
Since, by Theorem 2, $x-T x \in D(0) \subset D(C)$ for every $x \in E$ and all $T \in S$, it follows that $x^{*} \in F^{*}$ and hence

$$
\left\langle T_{\infty} x, x^{*}\right\rangle=\left\langle x, x^{*}\right\rangle=0 \quad(x \in D(C)) .
$$

Thus $x^{*}=0$, because $C=T_{\infty} D(C)$ separates $F^{*}$. This and the separation theorem imply that $D(C)=E$.

Conversely, suppose $T_{\infty} D=C$ and $D=E$. For an $x^{*} \in F^{*}$ with $x^{*} \neq 0$, choose an $x \in E$ so that $\left\langle x, x^{*}\right\rangle \neq 0$. Then we have

$$
\left\langle T_{\infty} x, x^{*}\right\rangle=\left\langle x, x^{*}\right\rangle \neq 0,
$$

which proves that $C=T_{\infty} D$ separates $F^{*}$. The proof is complete.

CoROLLARY 1. Let $E$ be a complete locally convex t.v.s. and $\subseteq$ a weakly right ergodic semigroup. Then the following conditions are equivalent:

(a) There exists a (unique) continuous linear operator $P$ on $E$ such that, for every $x \in E$ and all $T \in \mathfrak{S}$,

$$
P x \in \bar{A}(x) \text { and } P T=T P=P^{2}=P .
$$

(b) $E$ is the direct sum of $F$ and $N$, where $N$ is the closed linear subspace of $E$ determined by the set $\{x-T x: x \in E$ and $T \in \mathfrak{S}\}$.

(c) $F$ separates $F^{*}$.

(d) The set $\{x \in E: \bar{A}(x) \cap F \neq \varnothing\}$ is weakly dense in $E$.

Proof. $\quad(a) \Rightarrow(d)$ : Obvious.

$(d) \Rightarrow(c)$ : For an $x^{*} \in E^{*}$ with $x^{*} \neq 0$, take an $x \in E$ such that $\left\langle x, x^{*}\right\rangle \neq 0$. If $\mathrm{y} \in \bar{A}(x) \cap F$ then we have

$$
\left\langle y, x^{*}\right\rangle=\left\langle x, x^{*}\right\rangle \neq 0 .
$$

Hence the implication $(\mathrm{d}) \Rightarrow$ (c) follows.

(c) $\Rightarrow(\mathrm{b})$ : Let $\left(T_{\alpha}, \alpha \in \Lambda\right)$ be a weakly right $\varsigma_{\text {-ergodic net, and define }}$ $D$ and $T_{\infty}$ as in Theorem 1. It is then clear that $F=T_{\infty} F \subset T_{\infty} D$, therefore if $F$ separates $F^{*}$ then Theorem 3 implies that $T_{\infty} D=F$ and $D=E$. Now Theorem 2 implies that $T T_{\infty}=T_{\infty} T=T_{\infty}$ (on $E$ ) for all $T \in \mathcal{S}$. Therefore any $x \in E$ can be written as $x=T_{\infty} x+\left(x-T_{\infty} x\right)$, where $T_{\infty} x \in F$ and $x-T_{\infty} x \in D(0)=N$. Clearly $F \cap N=\{0\}$.

(b) $\Rightarrow($ a): Suppose $E$ is the direct sum of $F$ and $N$. Then, by Theorem 2, we have $D=E$. Hence, letting $P=T_{\infty}$ (on $E$ ), (a) follows. This completes the proof.

Corollary 2. Let $E$ and $\subseteq$ be as in Corollary 1. Then the following conditions are equivalent:

(a) For every $x \in E, 0 \in \bar{A}(x)$. 
(b) $E=N$.

(c) $F^{*}=\{0\}$.

(d) The set $\{x \in E: 0 \in \bar{A}(x)\}$ is weakly dense in $E$.

We omit the proof of Corollary 2.

Proposition 2. Let $E$ be a complete locally convex t.v.s. and $\mathfrak{S} a$ semigroup of continuous linear operators on E. Suppose that $\mathfrak{\subseteq}$ possesses a weakly left S-ergodic net $\left(T_{\alpha}, \alpha \in \Lambda\right)$ satisfying condition (S-I). If the set $\{T x: T \in \mathfrak{S}\}$ is relatively weakly compact in $E$, then $\bar{A}(x) \cap$ $F \neq \varnothing$.

Proof. Since $E$ is a complete locally convex t.v.s., Krein's theorem (cf. Theorem IV. 11.4 of [7]) implies that $\overline{c o} S_{x}$ is again weakly compact. On the other hand, we have $T(\overline{\mathrm{oc}} \subseteq x) \subset \overline{\mathrm{oc}} \subseteq x$ and weak- $\lim _{\alpha} T T_{\alpha} x-$ $T_{\alpha} x=0$ for every $T \in \mathfrak{S}$. Thus, it follows from an easy compactness argument that there exists an $x$ in $\overline{c o} \mathfrak{S} x$ which is a fixed point of $\mathfrak{S}$. This completes the proof.

\section{REFERENCES}

[1] M. M. DAY, Amenable semigroups, Illinois J. Math., 1 (1957), 509-544.

[2] M. M. DAY, Semigroups and amenability, Semigroups, (Proc. Symp., Wayne State Univ., Detroit, Mich., 1968), 5-53, Academic Press, Inc., New York, 1969.

[3] W. F. EberLein, Abstract ergodic theorems and weak almost periodic functions, Trans. Amer. Math. Soc., 67 (1949), 217-240.

[4] S. P. Lloyd, On the mean ergodic theorem of Sine, Proc. Amer. Math. Soc., 56 (1976), 121-126.

[5] R. J. NAGEL, Mittelergodische Halbgruppen linearer Operatoren, Ann. Inst. Fourier (Grenoble), 23-4 (1973), 75-87.

[6] W. Rudin, Functional Analysis, McGraw-Hill Book Co., New York, 1973.

[7] H. H. SchaEfER, Topological Vector Spaces, Springer-Verlag, New York-HeidelbergBerlin, 1971.

[8] R. Sine, A mean ergodic theorem, Proc. Amer. Math. Soc., 24 (1970), 438-439.

Department OF MATHEMATICS

JOSAI UNIVERSITY

SAKado, SAITAMa

350-02 JAPAN 
